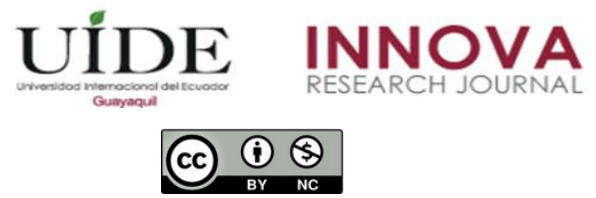

INNOVA Research Journal, ISSN 2477-9024

(Septiembre-Diciembre 2020). Vol. 5, No.3 pp. 103-121

DOI: https://doi.org/10.33890/innova.v5.n3.2020.1383

URL: http://revistas.uide.edu.ec/index.php/innova/index

Correo: innova@uide.edu.ec

\title{
Aplicación de Estrategias Investigativas y Desarrollo de Habilidades para elaborar Proyectos en Estudiantes Universitarios
}

\section{Application of Investigative Strategies and Development of Abilities to elaborate Projects in University Students}

\author{
Ilich Iván Pumacayo Palomino \\ https://orcid.org/0000-0003-1341-2613 \\ Universidad Privada César Vallejo, Perú \\ Kriss Melody Calla Vázquez \\ https://orcid.org/0000-0003-4976-332 \\ Judith Soledad Yangali Vicente \\ https://orcid.org/0000-0002-0759-645 \\ Claudia Milagros Arispe Alburqueque \\ https://orcid.org/0000-0003-0792-4655 \\ Universidad Privada Norbert Wiener, Perú
}

Autor para correspondencia: ilichpumacayop10@gmail.com; callavasquezkriss@ gmail.com; judith.yangali@uwiener.edu.pe; claudia.arispe@uwiener.edu.pe

Fecha de recepción: 30 de marzo del 2020 - Fecha de aceptación: 02 de junio del 2020

\section{Resumen}

Esta investigación formuló el objetivo de determinar la influencia de la aplicación de estrategias investigativas en las habilidades para elaborar proyectos de investigación. La metodología corresponde a los estudios de enfoque cuantitativo con un diseño cuasiexperimental. Se contó con una población de 145 estudiantes y con una muestra no probabilística distribuida en un grupo experimental (Administración de Empresas y Negocios Internacionales) 97, y grupo de control de 48 (Contabilidad). Para medir las variables se utilizó la técnica la encuesta y como instrumento el cuestionario, que fue validado mediante juicio de expertos y la confiabilidad Kuder - Richardson (0, 810 excelente confiabilidad). En los estadísticos descriptivos se halló que, en el pretest de las habilidades para elaborar proyectos de investigación, en el grupo experimental el 82, 5\% (80) tienen habilidades deficientes y en el grupo de control el 91, 7\% (44) tienen habilidades deficientes. En el postest de las habilidades para elaborar proyectos de investigación, se observa que en el grupo experimental el 52,6\% (51) tienen buenas habilidades y que el grupo de control el 68,8 \% (33) tienen habilidades deficientes. Como principal conclusión se indica que las aplicaciones de estrategias investigativas influyen significativamente en las habilidades para elaborar proyectos de investigación en estudiantes de la Facultad de Ciencias Empresariales de la Universidad César Vallejo, Ate, 2017. (En el Postest: $\mathrm{p}<0,05, \mathrm{Z}=-9,431>-1,96$ y rango promedio = 94,29 > 29,97). Palabras claves: habilidad; estrategias investigativas; deficiencia; estrategias; heurístico 


\begin{abstract}
This research formulated the objective of determining the influence of the application of investigative strategies in the abilities to develop research projects. The methodology corresponds to the studies of quantitative approach with a quasi-experimental design. There was a population of 145 students and a non-probabilistic sample distributed in an experimental group (Business Administration and International Business) 97, and a control group of 48 (Accounting). The survey technique was used to measure the variables and the questionnaire was used as an instrument, which was validated by expert judgment and the Kuder - Richardson reliability (0, 810 excellent reliability). In the descriptive statistics it was found that in the pretest of the skills to elaborate research projects, in the experimental group 82, 5\% (80) have poor skills and in the control group $91,7 \%$ (44) have skills deficient. In the post-test of the skills to elaborate research projects, it is observed that in the experimental group 52.6\% (51) have good skills and that the control group $68.8 \%$ (33) have poor skills. The main conclusion is that the application of research strategies significantly influences the skills to develop research projects in students of the Faculty of Business Studies of the César Vallejo University, Ate, 2017. (In the Posttest: $p<0.05, Z=-9,431\rangle$ -1.96 and average range $=94.29>29.97$ ).
\end{abstract}

Key words: skill; research strategies; deficiency; strategies; heuristic

\title{
Introducción
}

En la actualidad las universidades tienen el deber de fomentar la difusión y creación del conocimiento, esto se debe realizar mediante investigaciones exitosas que acarreen la renovación de contenidos académico y el impulso del progreso. La investigación pretende suministrar información acerca de la problemática de la producción de proyectos de investigación científica para obtener el título universitario. Al respecto existen estudios realizados a nivel internacional, tal es el caso de la universidad central Martha Abreu de las Villas en Cuba, Blanco (2016), realizó una investigación cuyo objetivo fue proponer un modelo teórico metodológico para desarrollar habilidades investigativas en el proceso docente con el fin de desarrollar dichas habilidades en los estudiantes de la carrera de medicina desde la educación en el trabajo, la integración de componentes organizacionales y personalizados, además de una orientación en las tareas investigativas elaboradas a partir del estudio de problemas de salud relacionados con su formación profesional.

En el Perú también se realizaron estudios de esta naturaleza, tal es el caso de Ruiz (2005), quien afirma en lo que concierne al nivel de disposición para realizar una tesis, los resultados muestran que, efectivamente, existe un nivel superior al moderado de disposición para la realización de una tesis. Esta afirmación se cumple en todos los factores que componen esta variable, por consiguiente, se podría concluir, que los estudiantes, en general consideran que la realización de una tesis es importante para su formación académica. En una investigación sustentada en la Universidad Mayor de San Marcos por Murcia (2015), tuvo como objetivo demostrar que, en el proceso de la enseñanza y el aprendizaje, la aplicación del método heurístico constituye un factor importante que contribuye al desarrollo de las habilidades investigativas en los estudiantes en etapa de investigación formativa. El estudio logró demostrar que al aplicar el método heurístico en la enseñanza de las habilidades para elaborar proyectos de investigación de los estudiantes mejoró en forma significativa evidenciándose en la motivación 
por la investigación en los estudiantes de contabilidad y auditoría de una universidad privada de Guayaquil.

A nivel local, en la Facultad de Ciencias Empresariales de la Universidad Cesar Vallejo, se ha detectado que existe una gran problemática para la elaboración de proyectos de investigación científica, esto por muchos factores, uno de los cuales se considera que es la motivación de logro, ya que hasta la fecha son poco los estudiantes de esta facultad que logran sustentar su tesis para obtener su título profesional.

El estudio se realizó a fin de responder a la problemática ¿Cómo influye la aplicación de estrategias investigativas en las habilidades para elaborar proyectos de investigación en estudiantes de la Facultad de Ciencias Empresariales de la Universidad Cesar Vallejo campus Ate cohorte 2018?, y para esto se analizaron datos tomados de 145 estudiantes en base a la aplicación de los procesos del análisis y resultados obtenidos, que puedan servir de soporte para futuras investigaciones y nuevas propuestas que contribuyan a mejorar la calidad educativa.

\section{La investigación científica}

La investigación científica es sistemática, metódica, cuyos conocimientos son expuestos a un procedimiento de experimentos y verificación. La indagación puede ser cualitativa o cuantitativa. Cuando la investigación es cualitativa, significa que se ha realizado una práctica ordenada para detallar un fenómeno o hecho social. Cuando la indagación es cuantitativa, significa que se ha desarrollado una línea metódica para aclarar un fenómeno o hecho natural.

Sáenz y Tinoco (1999) señalaron que las investigaciones deben de ser elaboradas por medio conocimientos adquiridos para implementar una búsqueda eficaz, es decir, para poder resolver las dudas presentes. No obstante, esto no es más que búsquedas constantes y certeras que tienen diversas caracterizaciones para conseguir una óptima investigación.

\section{Estrategias investigativas}

Posteriormente de ejecutar un programa de indagación, se debe cumplir diversas actividades que permitan relacionarse con las características de la incógnita, hecho o fenómeno, puesto que podemos llamar a la formación de actividades como un proyecto de investigación.

El proyecto del programa de indagación es un período del procedimiento investigativo. De este período depende la satisfacción. Las actividades permiten mejorar diversas estrategias que se explican a consiguientemente.

\section{Dimensiones de la variable estrategias investigativas}

"Es en algún sentido, una situación nueva o diferente de lo ya aprendido que requiere utilizar de modo estratégico técnicas ya conocidas" (Pozo \& Perez, 1998). 
En un problema se necesita de diversos procedimientos, ya que impide conseguir un equilibrado común. Puesto que, en este sentido, las fundamentales estrategias para reconocer una dificultad es conocer promover para prontamente concluir; es decir, reconocer las causas o circunstancias de la complicación, para determinar las consecuencias o efectos que provoca.

\section{Estrategias investigativas para desarrollar las bases teóricas y conceptuales}

Las bases teóricas y conceptuales forman parte del marco de información recogido por otros investigadores. Aunque muchos autores han mencionado que esta es la base de la investigación, en lo personal no concuerdo con ellos, ya que estos se han basado por lo recolectado en su propia investigación, dejando de lado lo hecho por muchos más investigadores.

Para desarrollar la parte teórica, quien investiga se guiará por una planificación y el cual tendrá que ejecutar una actividad donde podrá seleccionar la temática de la investigación y que esta corresponda idóneamente en función del debate que pueda presentarse, así como el rechazo o la ampliación de nuevos conocimientos, y ello dependerá del resultado arrojado por la investigación hecho y las estrategias tomadas para llegar a la misma.

\section{Estrategias investigativas para recopilar y seleccionar datos e información}

Galán (2011) señalo que para una investigación precisa y eficaz es necesario la implementación de recolección de datos, determinando así el tipo de recolección que será utilizado y las herramientas necesarias para la investigación. También es necesario puntualizar que los métodos de investigación se toman como medios o vías por el cual ayuda en la relación del que está investigando y el sujeto, y así poder llegar a lograr las metas que desean.

Las estrategias para reunir y elegir datos e investigación son habilidades subjetivas, propias del analista en las que sobresale la información. Son habilidades que el alumno adquiere y desarrolla en un trabajo de investigación. Le permite alcanzar a los datos que requiere para comprender y solucionar un conflicto de indagación.

\section{Definición de la variable}

En el primer grupo, Pérez y López (1999) definieron las habilidades para elaborar proyectos de investigación como un dominio de acciones que ayudan a regular las actividades, gracias a los conocimientos y costumbres que el investigador adquiera para realizar la búsqueda de los problemas existentes y también a las soluciones que puedan lograr.

Moreno, M. (2005), conceptualiza el significado del valor a la Zona de Desarrollo Próximo como lo siguiente:

Con la expresión habilidades para elaborar proyectos de investigación se hace referencia a un conjunto de habilidades de diversa naturaleza, que empiezan a desarrollarse desde antes de que el individuo tenga acceso a procesos sistemáticos de formación para la investigación, que en su mayoría no se desarrollan sólo para posibilitar la realización de 
las tareas propias de la investigación, pero que han sido detectadas por los formadores como habilidades cuyo desarrollo, en el investigador en formación o en funciones, es una contribución fundamental para potenciar que este pueda realizar investigación de buena calidad (p. 527).

Machado et al. (2008) definieron la habilidad para elaborar proyectos de investigación como "El dominio de la acción que se despliega para solucionar tareas investigativas en el ámbito docente, laboral y propiamente investigativo con los recursos de la metodología de la ciencia" (p. 164). Consideran que este concepto muestra las habilidades para elaborar proyectos de investigación como principal progreso sustantivo.

\section{Dimensiones de las habilidades para elaborar proyectos de investigación}

\section{Habilidades para elaborar el planteamiento del problema}

Según Chirino, V. (2002) estas habilidades se definen de la siguiente manera ya que los problemas existen en los niveles educativos, puesto que se entiende como la percepción de contradicciones primordiales en los contextos de profesionalización pedagógicas, por medio de la comparación de las realidades en la educación del investigador, a lo que lleva a identificar los problemas pedagógicos existentes.

El problema del contexto es el conocimiento de contradicciones esenciales en el argumento de representación profesional, mediante la asimilación de las circunstancias con los conocimientos científicos y valores ético-profesionales que tiene el sujeto, lo que conduce a la identificación de problemas profesionales.

En las contradicciones esenciales pueden señalarse las siguientes:

- Contradicciones ilustradas teóricamente que se manifiesta a partir de la escasez que existe para solucionar las insuficiencias del procedimiento y los obstáculos de la teoría que se domina para ello; es un desacuerdo entre el saber y el no saber.

- Contradicciones entre la hipótesis y el procedimiento, entre lo que se conoce y las nuevas exigencias del procedimiento, entre el saber y el saber hacer.

- Contradicciones ilustradas en las habilidades dadas entre el procedimiento cotidiano y la excelencia del egresado, entre el saber hacer y el deber ser.

El progreso de estas contradicciones esenciales lleva comprendido el razonamiento desde la hipótesis científica de la realidad, lo que permite determinar las condiciones contradictorias y reconocer el inconveniente profesional.

\section{Habilidades para elaborar las bases teóricas}

Según Chirino (2002), esta habilidad para teorizar la realidad educativa representa "la búsqueda, aplicación y socialización de los conocimientos científicos esenciales para interpretar 
y explicar la realidad educativa, así como asumir posiciones personales científicas y éticas que le permitan proyectarla de forma enriquecida" (p. 94).

A partir del análisis de las situaciones es necesario a darle una forma a la teoría, ya que toda información pertinente al caso de estudio va a ser fundamental para la comprensión del tema. Es decir, que recopilar la información será una base que establece el curso de la investigación, por lo que el investigador procederá a analizar todos los datos que tiene a su poder para poder aplicarlos, una vez realizado todo el proceso se obtendrán resultados que servirán de comparación para llegar a las debidas conclusiones, y así de ese modo proponer ciertas recomendaciones que podrán ayudar a los próximos investigadores que se interesen por un tema similar.

\section{Habilidades para elaborar el aspecto metodológico}

Según Chirino (2002), la habilidad para elaborar proyectos de investigación acerca de la realidad educativa se corresponde con la "verificación permanente del proceso y los resultados de la aplicación de propuestas educativas que constituyen alternativas científicas de solución a los problemas de la realidad educativa, lo que permite evaluar sus logros y dificultades desde posiciones científicas y éticas" (p. 94).

En la verificación del proceso es permitido ante todo que se evalúen los logros que se obtienen de la investigación, utilizando principalmente las ramas éticas y científicas. En de ese modo, dentro del análisis es posible que se constate la realidad, todo ello por medio del estudio de datos y gráficos, así como también la elaboración e implementación de instrumentos que permiten la recolección de los mismo, y que debido a su orden y a todo lo que indica se puede llegar a los resultados pertinentes y lograr hacer comparaciones justas con el objetivo que se planteó en el principio de la investigación.

\section{Metodología}

El enfoque utilizado en el presente trabajo de investigación fue cuantitativo. De acuerdo con Hernández, Fernández y Baptista (2014): “el enfoque cuantitativo usa la recolección de datos para probar hipótesis, con base en una medición numérica y el análisis estadístico, para establecer patrones de comportamiento y probar teorías".

El diseño de investigación tomado en cuenta fue el Cuasi experimental. Según Bernal (2006) "en este diseño el investigador utiliza usualmente grupos ya constituidos y puede ser diseños con un grupo de medición antes y después, diseños con grupos de comparación equivalente o diseños con series de tiempos interrumpidos" (p. 149). Con dos grupos: uno de control y otro experimental, el mismo que se grafica de la siguiente manera: 


\begin{tabular}{|cccc|}
\hline G.E. & 01 & X & 02 \\
G.C. & 03 & - & 04 \\
\hline
\end{tabular}

Dónde:

GE: Grupo experimental

GG: Grupo control

01 y 03: Pre- test

$\mathrm{X}: \quad$ Tratamiento Experimental (Aplicación de estrategias investigativas)

02 y 04: Post test

: No hay tratamiento experimental

El muestreo fue no probabilístico, pues la elección de la muestra depende de las características y los criterios de la investigación mas no de la probabilidad según la definición que da Hernández, Fernández y Baptista (2010). En consecuencia, la selección de ambos grupos fue por criterio del investigador. La población lo constituyeron 750 estudiantes, la muestra está conformada 145 estudiantes del noveno ciclo de la Facultad de Ciencias Empresariales: siendo 97 estudiantes de la escuela de Administración de Empresas y Negocios internacionales que constituyeron el Grupo Experimental y 48 estudiantes de la escuela de Contabilidad que conformaron el Grupo Control, de la Universidad Cesar Vallejo, del Campus Ate en el año 2018.

La técnica e instrumentos de recolección de datos de la investigación utilizadas en el trabajo de investigación fue la encuesta y el cuestionario respectivamente.

\section{Resultados}

Para la obtención de los resultados se administró una escala de Likert el cual medirá el nivel de estrategias investigativas para la elaboración de proyectos de investigación en los estudiantes universitarios.

Tabla. 1

¿Cuál es el porcentaje de habilidades en los grupos experimental y control? Distribución de frecuencias de las habilidades para elaborar proyectos de investigación en el pretest

\begin{tabular}{lccccc}
\hline \multirow{2}{*}{ Nivel } & \multirow{2}{*}{ Rangos } & \multicolumn{2}{c}{ Grupo experimental } & \multicolumn{2}{c}{ Grupo control } \\
\cline { 3 - 6 } & & $\mathbf{f}$ & $\mathbf{\%}$ & $\mathbf{f}$ & \% \\
\hline Excelente & {$[19-20]$} & 0 & $0,0 \%$ & 0 & $0,0 \%$ \\
Muy bueno & {$[17-18]$} & 0 & $0,0 \%$ & 0 & $0,0 \%$ \\
Bueno & {$[14-16]$} & 9 & $9,3 \%$ & 0 & $0,0 \%$ \\
Regular & {$[11-13]$} & 8 & $8,2 \%$ & 4 & $8,3 \%$ \\
Deficiente & {$[0-10]$} & 80 & $82,5 \%$ & 44 & $91,7 \%$ \\
Total & & 97 & & 48 & \\
\hline
\end{tabular}

Fuente: Elaboración propia 
En la tabla $\mathrm{N}^{\mathrm{o}} 1$, referentes a las habilidades para elaborar proyectos de investigación, observamos en el grupo experimental que el 82,5\% (80) tienen habilidades deficientes, otro 9,3\% (9) tienen buenas habilidades y otro $8,2 \%$ (8) tienen habilidades de nivel regular; en cambio, en el grupo de control el $91,7 \%$ (44) tienen habilidades deficientes y el 8,3\% (4) tienen habilidades de nivel regular.

Tabla. 2

Distribución de frecuencias de las habilidades para elaborar el planteamiento del problema en el pretest

\begin{tabular}{llcccc}
\hline \multirow{2}{*}{ Nivel } & Rangos & \multicolumn{2}{c}{ Grupo experimental } & \multicolumn{2}{c}{ Grupo control } \\
\cline { 2 - 5 } & & $\mathbf{f}$ & $\mathbf{\%}$ & $\mathbf{f}$ & \% \\
\hline Excelente & {$[10]$} & 0 & $0,0 \%$ & 0 & $0,0 \%$ \\
Muy bueno & {$[9]$} & 0 & $0,0 \%$ & 0 & $0,0 \%$ \\
Bueno & {$[7-8]$} & 17 & $17,5 \%$ & 0 & $0,0 \%$ \\
Regular & {$[5-6]$} & 44 & $45,4 \%$ & 5 & $10,4 \%$ \\
Deficiente & {$[0-4]$} & 36 & $37,1 \%$ & 43 & $89,6 \%$ \\
Total & & 97 & & 48 & \\
\hline
\end{tabular}

Fuente: Elaboración propia

En la tabla $\mathrm{N}^{\circ}$ 2, en el pretest referido a las habilidades para elaborar el planteamiento del problema, se observa que en el grupo experimental el 45,4\% (44) tienen habilidades de nivel regular, otro $37,1 \%$ (36) tienen habilidades deficientes y otro $17,5 \%$ (17) tienen habilidades buenas; por otro lado, en el grupo de control el 89,6\% (43) tienen habilidades deficientes y el $10,4 \%$ (5) tienen habilidades de nivel regular.

Tabla no 3

Las habilidades para elaborar las bases teóricas en el pretest

\begin{tabular}{llcccc}
\hline \multirow{2}{*}{ Nivel } & Rangos & \multicolumn{2}{c}{ Grupo experimental } & \multicolumn{2}{c}{ Grupo control } \\
\cline { 3 - 6 } & & $\mathbf{F}$ & $\mathbf{\%}$ & $\mathbf{f}$ & $\mathbf{\%}$ \\
\hline Excelente & {$[5]$} & 0 & $0,0 \%$ & 8 & $16,7 \%$ \\
Muy bueno & {$[4]$} & 2 & $2,1 \%$ & 10 & $20,8 \%$ \\
Bueno & {$[3]$} & 22 & $22,7 \%$ & 8 & $16,7 \%$ \\
Regular & {$[2]$} & 14 & $14,4 \%$ & 14 & $29,2 \%$ \\
Deficiente & {$[1]$} & 59 & $60,8 \%$ & 8 & $16,7 \%$ \\
Total & & 97 & & 48 & \\
\hline
\end{tabular}

Fuente: Elaboración propia

En la tabla $\mathrm{N}^{\mathrm{o}} 3$, en el pretest para evaluar las habilidades para elaborar las bases teóricas, se puede observar que en el grupo experimental el 60,8\% (59) tienen habilidades deficientes otro $22,7 \%$ (22) tienen buenas habilidades, un $14,4 \%$ tienen habilidades de nivel regular y un $2,1 \%$ (2) tienen habilidades muy buenas; por otro lado, en el grupo de control el 29,2\% (14) tienen habilidades de nivel regular, seguido por un 20,8\% (10) que tienen habilidades muy buenas, el 
$16,7 \%$ (8) tienen habilidades deficientes, otro $16,7 \%$ (8) tienen buenas habilidades y el 16,7\% (8) tienen habilidades excelentes.

Tabla no 4

Habilidades para elaborar el aspecto metodológico en el pretest

\begin{tabular}{llcccc}
\hline \multirow{2}{*}{ Nivel } & Rangos & \multicolumn{2}{c}{ Grupo experimental } & \multicolumn{2}{c}{ Grupo control } \\
\cline { 3 - 6 } & & F & \% & f & \% \\
\hline Excelente & {$[5]$} & 5 & $5,20 \%$ & 0 & $0,00 \%$ \\
Muy bueno & {$[4]$} & 4 & $4,10 \%$ & 2 & $4,20 \%$ \\
Bueno & {$[3]$} & 12 & $12,40 \%$ & 7 & $14,60 \%$ \\
Regular & {$[2]$} & 24 & $24,70 \%$ & 13 & $27,10 \%$ \\
Deficiente & {$[1]$} & 52 & $53,60 \%$ & 26 & $54,20 \%$ \\
Total & & 97 & & 48 & \\
\hline
\end{tabular}

Fuente: Elaboración propia a partir de los autores.

En la tabla $\mathrm{N}^{\circ}$ 4, en el pretest referido a las habilidades para elaborar el aspecto metodológico, se observa que en el grupo experimental el 53,6\% (52) tienen habilidades deficientes otro $24,7 \%$ (24) tienen habilidades de nivel regular, un $12,4 \%$ tienen buenas habilidades, el 5,2\% tienen habilidades excelentes y un 4,1\% (4) tienen habilidades muy buenas; por otro lado, en el grupo de control el 54,2\% (26) tienen habilidades de deficientes, seguido por $\mathrm{n}$ $27,1 \%$ (13) que tienen habilidades de nivel regular, el 14,6\% (7) tienen buenas habilidades, y el $4,2 \%$ (2) tienen habilidades muy buenas.

Tabla no 5

Distribución de frecuencias de las habilidades para elaborar proyectos de investigación en el postest

\begin{tabular}{lccccc}
\hline \multirow{2}{*}{ Nivel } & Rangos & \multicolumn{2}{c}{ Grupo experimental } & \multicolumn{2}{c}{ Grupo control } \\
\cline { 3 - 6 } & & $\mathbf{f}$ & $\mathbf{\%}$ & $\mathbf{f}$ & \% \\
\hline Excelente & {$[19-20]$} & 0 & $0,0 \%$ & 0 & $0,0 \%$ \\
Muy bueno & {$[17-18]$} & 11 & $11,3 \%$ & 0 & $0,0 \%$ \\
Bueno & {$[14-16]$} & 51 & $52,6 \%$ & 0 & $0,0 \%$ \\
Regular & {$[11-13]$} & 35 & $36,1 \%$ & 15 & $31,3 \%$ \\
Deficiente & {$[0-10]$} & 0 & $0,0 \%$ & 33 & $68,8 \%$ \\
Total & & 97 & & 48 & \\
\hline
\end{tabular}

Fuente: Elaboración propia

En la tabla 5, referidas al postest de las habilidades para elaborar proyectos de investigación, muestran que en el grupo experimental el 52,6\% (51) tienen buenas habilidades, otro $36,1 \%$ (35) tienen habilidades de nivel regular y otro 11,3\% (11) tienen habilidades muy buenas; por otro lado, en el grupo de control el 68,8\% (33) tienen habilidades deficientes y el $31,3 \%$ (15) tienen habilidades de nivel regular. 
Tabla $\mathbf{n}^{0} 6$

El postest de las habilidades para elaborar el planteamiento del problema en el postest

\begin{tabular}{llcccc}
\hline \multirow{2}{*}{ Nivel } & Rangos & \multicolumn{2}{c}{ Grupo experimental } & \multicolumn{2}{c}{ Grupo control } \\
\cline { 3 - 6 } & & F & \% & f & \% \\
\hline Excelente & {$[10]$} & 14 & $14,4 \%$ & 0 & $0,0 \%$ \\
Muy bueno & {$[9]$} & 22 & $22,7 \%$ & 0 & $0,0 \%$ \\
Bueno & {$[7-8]$} & 51 & $52,6 \%$ & 0 & $0,0 \%$ \\
Regular & {$[5-6]$} & 10 & $10,3 \%$ & 6 & $12,5 \%$ \\
Deficiente & {$[0-4]$} & 0 & $0,0 \%$ & 42 & $87,5 \%$ \\
Total & & 97 & & 48 & \\
\hline
\end{tabular}

Fuente: Elaboración propia

En la Tabla $\mathrm{N}^{\circ}$ 6, en el postest referido a las habilidades para elaborar el planteamiento del problema, se observa que en el grupo experimental el 52,6\% (51) tienen buenas habilidades, otro $22,7 \%$ (22) tienen habilidades muy buenas, el 14,4\% tienen habilidades excelentes y otro $10,3 \%$ (10) tienen habilidades de nivele regular; por otro lado, en el grupo de control el 87,5\% (42) tienen habilidades deficientes y el $12,5 \%$ (6) tienen habilidades de nivel regular.

Tabla n $\mathbf{n}^{0}$

Distribución de frecuencias de las habilidades para elaborar las bases teóricas en el postest

\begin{tabular}{llcccc}
\hline \multirow{2}{*}{ Nivel } & Rangos & \multicolumn{2}{c}{ Grupo experimental } & \multicolumn{2}{c}{ Grupo control } \\
\cline { 3 - 6 } & & $\mathbf{F}$ & $\mathbf{\%}$ & $\mathbf{F}$ & $\mathbf{\%}$ \\
\hline Excelente & {$[5]$} & 17 & $15,50 \%$ & 0 & $0,00 \%$ \\
Muy bueno & {$[4]$} & 37 & $38,10 \%$ & 8 & $16,70 \%$ \\
Bueno & {$[3]$} & 15 & $15,50 \%$ & 17 & $35,40 \%$ \\
Regular & {$[2]$} & 9 & $9,30 \%$ & 12 & $25,00 \%$ \\
Deficiente & {$[1]$} & 19 & $19,60 \%$ & 11 & $22,90 \%$ \\
Total & & 97 & & 48 & \\
\hline
\end{tabular}

Fuente: Elaboración propia

En la tabla $\mathrm{N}^{\circ} 7$, del postest para evaluar las habilidades para elaborar las bases teóricas, se observa que en el grupo experimental el 35,1\% (34) tienen buenas habilidades otro 28,9\% (28) tienen habilidades de nivel regular, un 16,5\% (16) tienen habilidades excelentes, el 15,5\% (15) tienen habilidades buenas y un 4,1\% (4) tienen habilidades deficientes; por otro lado, en el grupo de control el 29,2\% (14) tienen buenas habilidades otro 27,1\% (13) tienen habilidades excelentes, un 22,9 (11) tienen habilidades muy buenas, el 16,7\% (8) tienen habilidades de nivel regular y un 4,2\% (2) tienen habilidades deficientes. 
Tabla $\mathbf{n}^{0} 8$

Distribución de frecuencias de las habilidades en el aspecto metodológico en el postest

\begin{tabular}{llcccc}
\hline \multirow{2}{*}{ Nivel } & Rangos & \multicolumn{2}{c}{ Grupo experimental } & \multicolumn{2}{c}{ Grupo control } \\
\cline { 3 - 6 } & & F & \% & f & \% \\
\hline Excelente & {$[5]$} & 16 & $16,50 \%$ & 13 & $27,10 \%$ \\
Muy bueno & {$[4]$} & 15 & $15,50 \%$ & 11 & $22,90 \%$ \\
Bueno & {$[3]$} & 34 & $35,10 \%$ & 14 & $29,20 \%$ \\
Regular & {$[2]$} & 28 & $28,90 \%$ & 8 & $16,70 \%$ \\
Deficiente & {$[1]$} & 4 & $4,10 \%$ & 2 & $4,20 \%$ \\
Total & & 97 & & 48 & \\
\hline
\end{tabular}

En la tabla $\mathrm{N}^{\circ}$ 8, en el postest para evaluar las habilidades para elaborar el aspecto metodológico, se observa que en el grupo experimental el 38,1\% (37) tienen habilidades muy buenas, otro $19,6 \%$ (24) tienen habilidades deficientes, un 15,5\% (17) tienen habilidades excelentes, el 15,5\% (15) tienen buenas habilidades y un 9,3\% (9) tienen habilidades de nivel regular; por otro lado, en el grupo de control el 35,4\% (17) tienen buenas habilidades, seguido por un 25\% (12) que tienen habilidades de nivel regular, el 22,9\% (11) tienen habilidades deficientes.

Tabla no 9

Prueba estadística para la determinación de la normalidad

\begin{tabular}{lccc}
\hline & \multicolumn{3}{c}{ Kolmogorov-Smirnov } \\
& Estadístico & gl & Sig. \\
\hline $\begin{array}{l}\text { Habilidades para elaborar proyectos de investigación } \\
\text { Pretest }\end{array}$ &, 129 & 145 &, 000 \\
$\begin{array}{l}\text { Habilidades para elaborar proyectos de investigación } \\
\text { Postest }\end{array}$ &, 123 & 145 &, 000 \\
\hline
\end{tabular}

Corrección de significación de Lilliefors

Fuente: Elaboración propia

En la tabla $N^{o}$ 9, dado que p < 0,05 (0,000 y 0,000); rechazamos la Hipótesis nula y aceptamos la Hipótesis alterna. Esto significa, según lo evidenciado que los datos de la muestra de estudio no provienen de una distribución normal. Asimismo, se observa en los gráficos siguientes la curva de distribución difiere de la curva normal. 


\section{Figura $\mathbf{n}^{0} 1$}

Distribución de frecuencias de los puntajes de la prueba de Habilidades para elaborar proyectos de investigación en el pretest

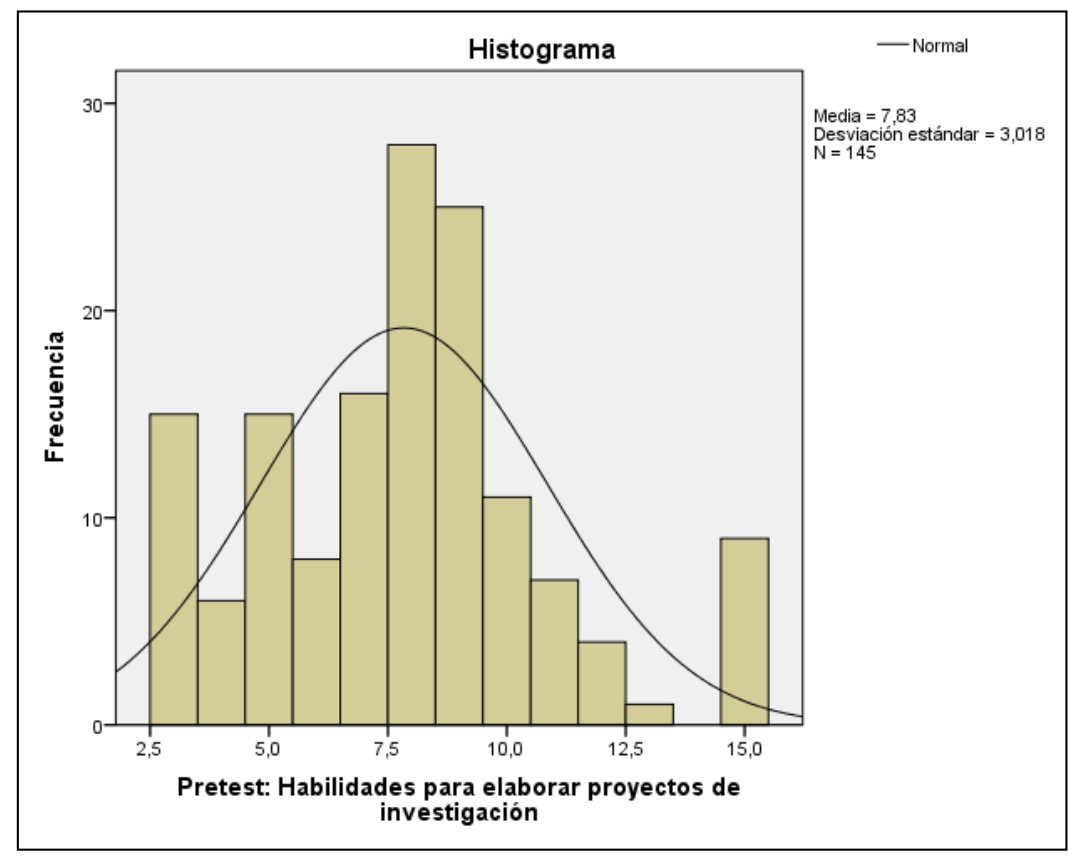

Según se aprecia en la Figura1, la distribución de frecuencias de los puntajes obtenidos en la prueba de Habilidades para elaborar proyectos de investigación en el pretest, sesgados hacia la derecha, teniendo una media de 7,83 y una desviación estándar de 3,018, asimismo, el gráfico muestra que la distribución de los datos difiere de la curva normal. 


\section{Figura $\mathbf{n}^{0} 2$}

Distribución de frecuencias de los puntajes de la prueba de Habilidades para elaborar proyectos de investigación en el postest

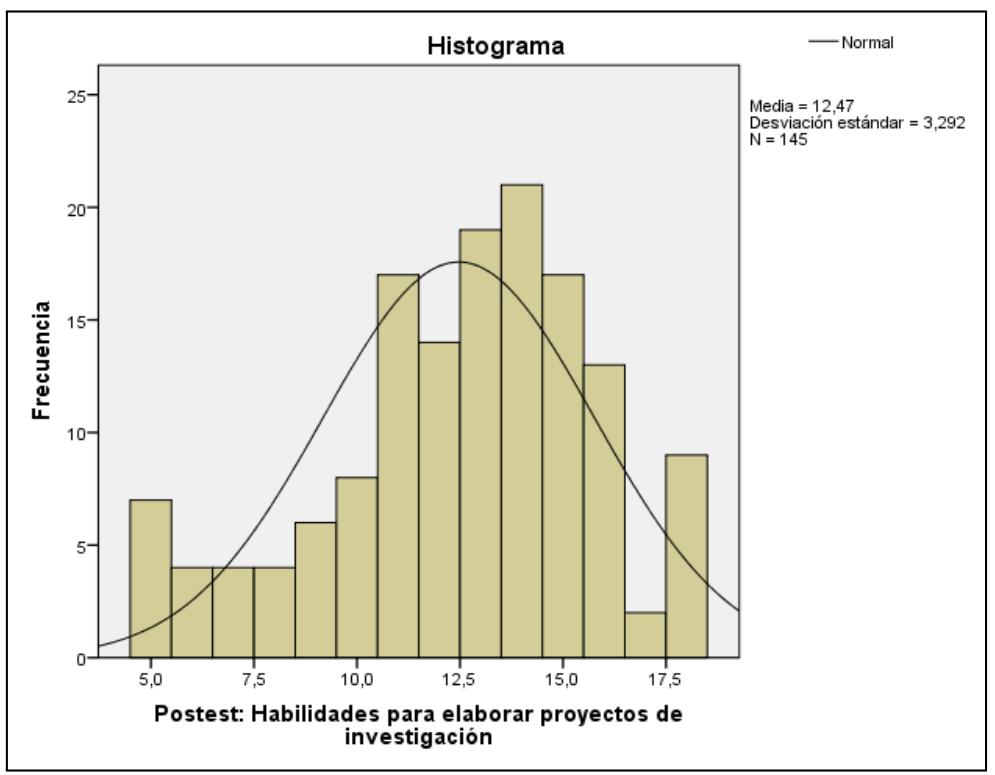

Se puede apreciar en la Figura 2, la distribución de frecuencias de los puntajes obtenidos a través de la prueba de habilidades para elaborar proyectos de investigación en el postest se encuentra sesgados hacia la derecha, teniendo una media de 12,47 y una Desviación estándar de 3,292, además, la figura muestra que los datos no tienen una distribución normal. Así mismo, se observa que el nivel de significancia (Sig. asintót. (bilateral) para Kolgomorov-Smirnov es menor a 0,05 tanto en los puntajes obtenidos a nivel de la prueba de Habilidades para elaborar proyectos de investigación en el pretest como en el postest. Hernández, Fernández y Baptista (2014) "se deduce que la distribución de estos puntajes en ambos casos difiere de la distribución normal, por lo tanto, para el desarrollo de la prueba de hipótesis; se utilizarán las pruebas no paramétricas para distribución normal de los datos U de Mann-Whitney” (p. 325).

Tabla $\mathbf{n}^{\circ} 10$

Prueba U de Mann-Whitney para muestras independientes

\begin{tabular}{lll}
\hline & $\begin{array}{l}\text { Pretest: Habilidades para elaborar } \\
\text { proyectos de investigación }\end{array}$ & $\begin{array}{l}\text { Postest: Habilidades para elaborar } \\
\text { proyectos de investigación }\end{array}$ \\
\hline U de Mann-Whitney & 2096,000 & 262,500 \\
Z & $-1,594$ & $-9,131$ \\
Sig. asintótica (bilateral) &, 111 &, 000 \\
a. Variable de agrupación: Grupos & \\
\hline
\end{tabular}

Fuente: Elaboración propia a partir de los autores.

La tabla $\mathrm{N}^{\mathrm{o}} 10$ presenta la comparación entre el grupo de control y grupo experimental antes y después de la aplicación de la aplicación de las estrategias investigativas. Los resultados de la prueba U de Mann Whitney en la condición pre test indican la no existencia de diferencias significativas en los niveles de las habilidades para elaborar proyectos de investigación entre los grupos de estudio en el pretest $(z=-0,1,594, p>0.01)$, siendo mayor el rango promedio en el 
grupo de experimental $(75,39)$ con respecto al grupo control (rango promedio $=68,17$ ); pero luego de la aplicación de la aplicación de las estrategias investigativas (post test) se observa diferencias altamente significativas $(\mathrm{z}=-9,131, \mathrm{p}<0.01)$ a favor del grupo experimental, porque el rango promedio del grupo experimental $(94,29)$ mayor a la del grupo de control $(29,97)$.

Por tanto, sobre la base de los resultados obtenidos se rechaza la hipótesis nula, en consecuencia, se acepta la hipótesis alterna la cual sostiene que: La aplicación de estrategias investigativas influyen significativamente en las habilidades para elaborar proyectos de investigación en estudiantes de la Facultad de Ciencias Empresariales de la Universidad César Vallejo, Ate, 2018.

\section{Tabla no 11}

\section{Prueba U de Mann-Whitney para muestras independientes}

\begin{tabular}{lll}
\hline & $\begin{array}{l}\text { Pretest: Habilidades para realizar el } \\
\text { planteamiento del problema }\end{array}$ & $\begin{array}{l}\text { Postest: Habilidades para realizar el } \\
\text { planteamiento del problema }\end{array}$ \\
\hline U de Mann-Whitney & 1064,000 & 30,000 \\
Z & $-1,945$ & $-10,031$ \\
Sig. asintótica (bilateral) &, 451 &, 000 \\
a. Variable de agrupación: Grupos & \\
\hline Fuente: Elaboración propia a partir de los autores.
\end{tabular}

Fuente: Elaboración propia a partir de los autores.

La tabla 11 presenta la comparación entre el grupo de control y grupo experimental antes y después de la aplicación de la aplicación de las estrategias investigativas. Los resultados de la prueba U de Mann Whitney en la condición pre test indican la no existencia de diferencias significativas en los niveles de las habilidades para realizar el planteamiento del problema entre los grupos de estudio en el pretest $(\mathrm{z}=-0,194, \mathrm{p}>0.01)$, siendo mayor el rango promedio en el grupo de experimental $(86,03)$ con respecto al grupo control (rango promedio $=46,67$ ); pero luego de la aplicación de la aplicación de las estrategias investigativas (post test) se observa diferencias altamente significativas $(\mathrm{z}=-10,031, \mathrm{p}<0.01)$ a favor del grupo experimental, porque el rango promedio del grupo experimental $(96,69)$ mayor a la del grupo de control $(25,13)$.

Por tanto, sobre la base de los resultados obtenidos se rechaza la hipótesis nula, en consecuencia, se acepta la hipótesis alterna la cual sostiene que: La aplicación de estrategias investigativas influyen significativamente en las habilidades para realizar el planteamiento del problema en estudiantes de la Facultad de Ciencias Empresariales de la Universidad César Vallejo, Ate, 2018.

\section{Tabla no 12}

Prueba U de Mann-Whitney para muestras independientes

\begin{tabular}{lll}
\hline & $\begin{array}{l}\text { Pretest: Habilidades para realizar las } \\
\text { bases teóricas }\end{array}$ & $\begin{array}{l}\text { Postest: Habilidades para realizar las } \\
\text { bases teóricas }\end{array}$ \\
\hline U de Mann-Whitney & 1088,000 & 1856,500 \\
$\mathrm{Z}$ & $-1,539$ & $-5,539$ \\
Sig. asintótica (bilateral) &, 000 &, 000 \\
a. Variable de agrupación: Grupos & \\
\hline Fuente: Elaboración propia a partir de los autores
\end{tabular}


La tabla 12 presenta la comparación entre el grupo de control y grupo experimental antes y después de la aplicación de las estrategias investigativas. Los resultados de la prueba U de Mann Whitney en la condición pre test indican la no existencia de diferencias significativas en los niveles de las habilidades para realizar las bases teóricas entre los grupos de estudio en el pretest $(\mathrm{z}=-1,539, \mathrm{p}>0.01)$, siendo mayor el rango promedio en el grupo de experimental $(60,22)$ con respecto al grupo control (rango promedio $=98,83)$; pero luego de la aplicación de la aplicación de las estrategias investigativas (post test) se observa diferencias altamente significativas $(\mathrm{z}=-5,539, \mathrm{p}<0.01)$ a favor del grupo experimental, porque el rango promedio del grupo experimental $(82,82)$ mayor a la del grupo de control $(68,14)$.

Por tanto, sobre la base de los resultados obtenidos se rechaza la hipótesis nula, en consecuencia, se acepta la hipótesis alterna la cual sostiene que: La aplicación de estrategias investigativas influyen significativamente en las habilidades para realizar las bases teóricas en estudiantes de la Facultad de Ciencias Empresariales de la Universidad Cesar Vallejo, Ate, 2018.

Tabla n' 13

Prueba U de Mann-Whitney para muestras independientes

\begin{tabular}{lll}
\hline & $\begin{array}{l}\text { Pretest: Habilidades para realizar el } \\
\text { aspecto metodológico }\end{array}$ & $\begin{array}{l}\text { Postest: Habilidades para realizar el } \\
\text { aspecto metodológico }\end{array}$ \\
\hline U de Mann-Whitney & 2262,000 & 1482,000 \\
$\mathrm{Z}$ &,- 305 & $-3,655$ \\
Sig. asintótica (bilateral) & 0,760 &, 000 \\
a. Variable de agrupación: Grupos & \\
\hline \multicolumn{2}{l}{ Fuente: Elaboración propia a partir de los autores. }
\end{tabular}

. La tabla 13 presenta la comparación entre el grupo de control y grupo experimental antes y después de la aplicación de la aplicación de las estrategias investigativas. Los resultados de la prueba U de Mann Whitney en la condición pre test indican la no existencia de diferencias significativas en los niveles de las habilidades para realizar el aspecto metodológico entre los grupos de estudio en el pretest $(\mathrm{z}=-, 305, \mathrm{p}>0.01)$, siendo mayor el rango promedio en el grupo de experimental $(73,68)$ con respecto al grupo control (rango promedio $=71,63$ ); pero luego de la aplicación de la aplicación de las estrategias investigativas (post test) se observa diferencias altamente significativas $(\mathrm{z}=-3,655, \mathrm{p}<0.01)$ a favor del grupo experimental, porque el rango promedio del grupo experimental $(81,72)$ mayor a la del grupo de control $(55,38)$.

Por tanto, sobre la base de los resultados obtenidos se rechaza la hipótesis nula, en consecuencia, se acepta la hipótesis alterna la cual sostiene que: La aplicación de estrategias investigativas influyen significativamente en las habilidades para realizar el aspecto metodológico en estudiantes de la Facultad de Ciencias Empresariales de la Universidad Cesar Vallejo, Ate, 2018.

\section{Discusión de resultados}

Luego del análisis de los resultados se logró hallar que la aplicación de estrategias investigativas que influyen significativamente en las habilidades para elaborar proyectos de investigación en estudiantes de la Facultad de Ciencias Empresariales de la Universidad Cesar 
Vallejo, Ate, 2018. (En el Postest: $\mathrm{p}<0,05, \mathrm{Z}=-9,431>-1,96$ y Rango promedio = 94,29 > 29,97), al respecto se hallaron resultados similares en la tesis de Herrera (2016), titulada Estrategias investigativas y su influencia en la elaboración del proyecto de investigación científica. Caso: estudiantes de la Facultad de Ciencias Políticas y Administrativas de la Universidad Nacional de Chimborazo, Riobamba en Ecuador - 2015, donde se ha demostrado que existe una influencia muy significativa del programa de estrategias investigativas PEIPIC en el aprendizaje de la planificación del proyecto de investigación científica, según Mann Whitney de 0,000 y siendo altamente significativo.

Por su parte Murcia (2015), en su tesis Propuesta didáctica para desarrollar competencias investigativas en estudiantes de carreras técnicas profesionales en el centro de investigación, docencia y consultoría administrativa- CIDCA- Bogotá, evidenció que hay una deficiencia en la comprensión de lectura y en la redacción, es decir que independientemente de lo que se trabaje en investigación, los estudiantes requieren de un refuerzo en lectoescritura, pertinentes en otras asignaturas establecidas en su plan de estudios, también que los talleres y documentos presentados en clase, en grupo debería reflejar un mejor desempeño, por tener la participación de varias personas, pero por el contrario esto lleva a pensar, que cuando se conforman equipos de estudio, con frecuencia no evidencian la efectividad requerida y se asocian solo para cumplir con el requisito de la asignatura, sin reflejarse aportes en las actividades y alcance de las competencias definidas para la materia, esto se refleja al momento de exponerle a docente.

Por otro lado, se halló que las aplicaciones de estrategias investigativas influyen significativamente en las habilidades para realizar el planteamiento del problema en estudiantes de la Facultad de Ciencias Empresariales de la Universidad César Vallejo, Ate, 2018. (En el Postest: $\mathrm{p}<0,05, \mathrm{Z}=-10,031>-1,96$ y Rango promedio $=96,69>25,13$ ), al respecto se hallaron resultados similares en la tesis de Quipuscoa (2013), titulada Modelo matricial para la evaluación de la consistencia epistémica del discurso científico de informes de tesis de egresados del Instituto Superior Pedagógico Público "Indoamérica" de Trujillo-Perú, donde se concluyó que el nivel de consistencia epistemológica del discurso científico de los informes de tesis de los egresados del instituto Superior Pedagógico Público "Indoamérica" de Trujillo fue Medio-Bajo en las especialidades de Educación Inicial y Secundaria; en la especialidad de Educación Primaria, la cuarta parte de informes alcanzó un nivel Óptimo en las dimensiones de coherencia, completitud, alcance explicativo y pertinencia; ninguno de los discursos.

Asimismo, se encontró que las aplicaciones de estrategias investigativas influyen significativamente en las habilidades para realizar las bases teóricas en estudiantes de la Facultad de Ciencias Empresariales de la Universidad César Vallejo, Ate, 2018. (En el Postest: p < 0,05, Z $=-5,539>-1,96$ y Rango promedio $=82,82>68,14)$, al respecto se hallaron resultados similares en la tesis de Ordoñez (2017), titulata Aplicación del método heurístico y desarrollo de habilidades de investigación en estudiantes en etapa de investigación formativa, donde concluyó que al validar la estrategia didáctica metodológica con base en el método heurístico que guíe el desarrollo de las habilidades de investigación se demuestra la relación causal existente entre la aplicación del método heurístico y el desarrollo de habilidades de investigación en los estudiantes en etapa formativa de la Carrera de Contabilidad y Auditoría de una universidad privada de la ciudad de Guayaquil. 
Por último, se halló que las aplicaciones de estrategias investigativas influyen significativamente en las habilidades para realizar el aspecto metodológico en estudiantes de la Facultad de Ciencias Empresariales de la Universidad Cesar Vallejo, Ate, 2018. (En el Postest: p $<0,05, Z=-3,655>-1,96$ y Rango promedio $=81,72>55,38)$, al respecto se hallaron resultados similares Figueroa (2017), titulada Estrategia de aprendizaje para desarrollar habilidades investigativas en los estudiantes de la Escuela de Cultura Física de la Universidad Técnica de Babahoyo, donde se concluyó que los talleres pedagógicos como estrategia de aprendizaje han influido de forma significativa en el Desarrollo de Habilidades investigativas en los estudiantes, porque se aprecian diferencias significativas entre los grupos de control y experimental en el post test de las habilidades investigativas, según la $U$ de Mann-Whitney, siendo el nivel de significancia de $95 \%$ menor al 0,05 es decir ,000 por lo que se rechaza la hipótesis nula y se tiene que: Existe una diferencia significativa entre el grupo de control y el grupo experimental en el post test, luego de haberse aplicado los talleres pedagógicos como estrategia de aprendizaje en el desarrollo de las habilidades investigativas de los estudiantes.

\section{Conclusiones}

La aplicación de estrategias investigativas influye significativamente en las habilidades para elaborar proyectos de investigación en estudiantes de la Facultad de Ciencias Empresariales de la Universidad Cesar Vallejo, Ate, 2018

Las aplicaciones de estrategias investigativas influyen significativamente en las habilidades para realizar el planteamiento del problema en estudiantes de la Facultad de Ciencias Empresariales de la Universidad Cesar Vallejo, Ate, 2018

Las aplicaciones de estrategias investigativas influyen significativamente en las habilidades para realizar las bases teóricas en estudiantes de la Facultad de Ciencias Empresariales de la Universidad Cesar Vallejo, Ate, 2018.

Las aplicaciones de estrategias investigativas influyen significativamente en las habilidades para realizar el aspecto metodológico en estudiantes de la Facultad de Ciencias Empresariales de la Universidad Cesar Vallejo, Ate, 2018.

\section{Bibliografía}

Bernal, C. (2006). Metodologia De La Investigacion. Colombia: Pearson.

Blanco, N. (2016). El desarrollo de las habilidades investigativas en los estudiantes de medicina desde la educación en el trabajo (Tesis doctoral). Cuba: Universidad Central Marta Abreu de las Villas.

Camposeco, F. (2012). La autoeficacia como variable en la motivación intrínseca y extrínseca en matemáticas a través de un criterio étnico. (Tesis doctoral). Universidad Complutense de Madrid, España. Recuperado de: http://eprints.ucm.es/16670/1/T34002.pdf

Carrasco, S. (2009) Metodología de la investigación científica. Lima: Editorial San Marcos.

Chirino, V. (2002). Perfeccionamiento de la formación inicial investigativa de los profesionales de la educación. (Tesis Doctoral), Instituto Superior Pedagógico "Enrique José Varona", La Habana. 
Dewey, J. (1989). Cómo pensamos. España: Paidós.

Fernández, C. (2012) Metodología Docente, Motivación Y Rendimiento. (Tesis doctoral). Universidad Autónoma de Madrid. Madrid.

Figueroa, M. (2017). Estrategia de aprendizaje para desarrollar habilidades investigativas en los estudiantes de la Escuela de Cultura Física de la Universidad Técnica de Babahoyo. Lima: Universidad Nacional Mayo de San Marcos. Obtenido de http://cybertesis.unmsm.edu.pe/bitstream/handle/cybertesis/6965/Figueroa_sm.pdf?sequ ence $=1$

Flores, J. (1993) Investigación científica. Lima: UNMSM

Galán, M. (2011). Metodología de la Investigación. Obtenido de Definición de políticas y líneas de investigación en la Universidad: http://manuelgalan.blogspot.com

Gonález, T. (2017). Modelo para el desarrollo de competencias investigativas con enfoque interdisciplinario en tecnología de la Salud (Tesis doctoral). Cuba: Universidad de Ciencias Médicas de la Habana. Obtenido de file://C:/Users/Marcos/Downloads/Tania\%20Rosa.pdf

Herrera, C. (2016). Estrategias investigativas y su influencia en la elaboración del proyecto de investigación científica. Caso: estudiantes de la Facultad de Ciencias Políticas y Administrativas de la Universidad Nacional de Chimborazo. Riobamba, Ecuador. 2015. Lima: Universidad Nacional Mayor de San Marcos.

Linares, J. (2014). Estudio de la motivación al logro en los estudiantes de la escuela de bibliotecología y archivología de la UCV. (Tesis de Maestría). Venezuela: Universidad Central de Venezuela. Recuperado de: http://saber.ucv.ve/bitstream/123456789/1253/1/MOTIVACI.PDF

Machado Ramírez, E. F., Montes de Oca Recio, N., \& Mena Campos, A. (2008). El desarrollo de habilidades investigativas como objetivo educativo en las condiciones de la universalización de la educación superior. Pedagogía Universitaria. XIII (1), 156-180. Recuperado http://revistas.mes.edu.cu/PedaQOQiaUniversitaria/articulos/2008/numero/189408108.p df

Moreno, M.G. (2005). Potenciar la educación. Un curriculum transversal de formación para la investigación. Revista Electrónica Iberoamericana sobre Calidad, Eficacia y Cambio en Educación, $\quad 3 \quad(1), \quad 520-540 . \quad$ Recuperado de http://dialnet.unirioja.es/servlet/articulo?codiqo=1130331

Murcia, J. (2015). Propuesta didáctica para desarrollar competencias investigativas en estudiantes de carreras técnicas profesionales en el centro de investigación, docencia y consultoría administrativa- CIDCA- Bogotá. Bogotá: Universidad Militar Nueva Granada.

Nagamine, M. (2017). Factores para el logro de las competencias investigativas en una universidad privada, Lima 2015. Lima: Universidad Cesar Vallejo.

Naupas, H. (2011) Metodología de la Investigación Científica y Asesoramiento de Tesis Segunda Edición. Lima, Perú: Universidad Nacional Mayor de San Marcos.

Ordoñez, R. (2017). Aplicación del método heurístico y desarrollo de habilidades de investigación en estudiantes en etapa de investigación formativa. (Tesis doctoral) Universidad Nacional Mayor de San Marcos. Lima.

Pérez, C. \& López, L. (1999). Las habilidades e invariantes investigabas en la formación del profesorado. Una propuesta metodológica para su estudio. Pedagogía Universitaria, 
4(2), 13-44. Recuperado de http://revistas.mes.edu.cu/PedaqoqiaUniversitaha/articulos/1999/2/189499202.pdf

Pozo, I. y Pérez, P. (1998) La solución de problemas. Aula XXI. México: Santillana.

Quipuscoa, M. (2013). Modelo matricial para la evaluación de la consistencia epistémica del discurso científico de informes de tesis de egresados del Instituto Superior Pedagógico Público "Indoamérica” de Trujillo-Perú. Trujillo: Universidad Nacional de Trujillo.

Robles, J. (2013). Aplicación de estrategias didácticas para la formación de competencias investigativas en niñas y niños del cuarto grado de la EScuela Dr. Carlos Roberto Reina del Municipio de Trojes, Departamento de el Paraiso (Tesis de Maestría). Honduras: Universidad Pedagógica Nacional Francisco Morazán. Obtenido de file://C:/Users/Marcos/Downloads/aplicacion-de-estrategias-didactias-para-laformacion-de-competencias-investigativas-en-ninos-y-ninas-del-cuarto-grado-de-laescuela-dr-carlos-roberto-reina-del-municipio-de-trojes-departamento-de-elparaiso\%20(1).pdf

Ruiz, F. (2005). Relación entre la motivación de logro académico, la autoeficacia y la disposición para la realización de una tesis. Universidad de Lima. Recuperado de: https://revistas.ulima.edu.pe/index.php/Persona/article/view/898

Sáenz, D. y Tinoco, S. (1999) Introducción a la investigación científica. CENDEISSS.

Valderrama, S. (2010) Metodología del trabajo universitario. Perú: San Marcos

Villegas, L., Marroquín, R. Del Castillo, V. y Sánchez, R. (2011). Teoría y práxis de la investigación científica. Tesis de maestría y doctorado. Lima: Editorial San Marcos. 